\title{
Elementos para fundamentar políticas públicas para la población adulta mayor
}

\section{Aspects to substantiate public policies to the elderly people population}

\begin{abstract}
José Manuel Salazar ${ }^{1}$
${ }^{1}$ Maestría en Análisis y Evaluación de Proyectos; Profesor, Universidad de Panamá, Centro Regional Universitario de Veraguas; jose.salazara@up.ac.pa; https://orcid.org/0000-0001-9923-9350

Resumen: El envejecimiento de la población, es entendido como un "fenómeno debido al cual las personas de más edad representan una parte proporcionalmente mayor del total de la población" (ONU, 2014). El incremento en la esperanza de vida y la baja tasa de natalidad han dado como consecuencia que en muchos países se dé un crecimiento en la población de adultos mayores. Para que esta población tenga un envejecimiento saludable se requiere que los Estados promuevan estrategias y planes de acción que incorporen aspectos sociales, económicos y políticos. El artículo tiene como objetivo señalar que la vejez no es una enfermedad, sino que es una etapa para la cual se requieren desarrollar políticas públicas. Además, el artículo presenta que los indicadores de envejecimiento de la población muestran una gran heterogeneidad entre países, que la dinámica de la población ha variado.
\end{abstract}

Palabras clave: adultos mayores, políticas públicas, planificación.

\begin{abstract}
Population aging is understood as a phenomenon in which elder people represent a major percentage over the entire population (ONU, 2014). An increasing life expectancy and a lower birth rate have caused an increment of the elderly population. For a healthier aging lifestyle, it is required that every country develops new policies and strategies which incorporate political, economic, and social aspects. Old age is not a disease, but it is a stage that needs public policies. Besides, this article presents elderly population indicators which display considerable diversity to the aging population among countries and that the population behavior has changed throughout time.
\end{abstract}

Key words: elder people, public policies, planning.

\section{Introducción}

La población mundial está envejeciendo: la mayoría de los países del mundo están experimentando un aumento en el número y la proporción de personas mayores. Según las estimaciones y proyecciones de las Naciones Unidas, en 2019 había 84,9 millones de personas mayores en América Latina y el Caribe, cifra que representa el 13\% de la población regional. En 2030 esta población ascenderá a 118 millones y constituirá el 16,7\% de la 
población total. En 2050, cuando el envejecimiento esté presente en prácticamente todos los países de la región, habrá alrededor de 190 millones de personas mayores, que representarán el 25\% de la población regional (CEPAL, 2019), (ver figura 1). Este proceso acelerado de envejecimiento que presentan muchos países incluyendo Panamá tiene efecto en todas las dimensiones de la vida por lo que obliga contar un marco jurídico actualizado y con instituciones públicas y privadas que garanticen estrategias en la calidad de vida del adulto mayor con acciones de promoción y prevención.

Figura 1. América Latina y el Caribe: número de personas de 60 años y más, 2017-2060.

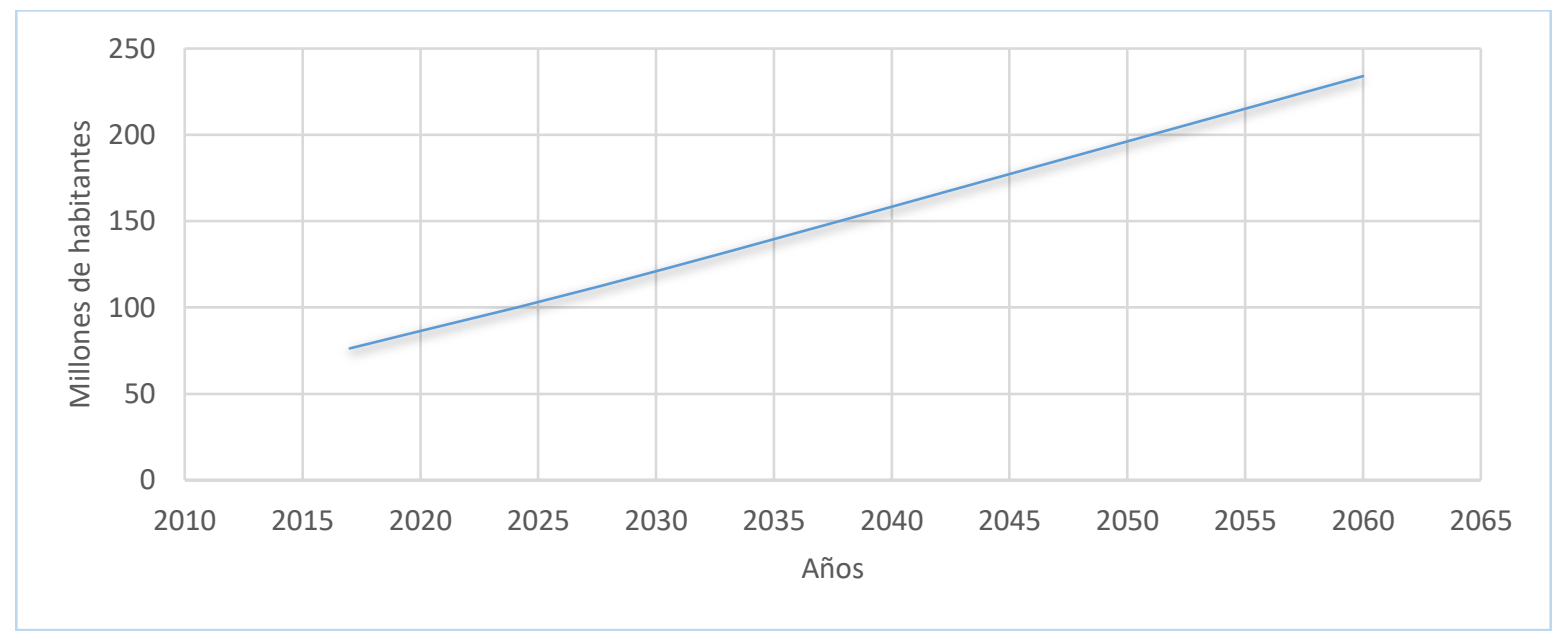

Fuente: Naciones Unidas, "World Population Prospects: The 2015 Revision. Key findings and Advance Tables", Working Paper, N² 241 (ESA/P/WP.24), Nueva York, 2015.

Este envejecimiento se debe a muchos factores como la baja fecundidad, en décadas pasadas las tasas de fecundidad fueron altas (5.5 hijos por mujer) pero ahora la tasa de fecundidad ha bajado (2.05 hijos por mujer). En Panamá el índice de envejecimiento aumentará 69.9\% en 2030.

Como se señaló estos cambios demográficos del siglo XX se vieron afectados por una disminución constante de las tasas de fertilidad y mortalidad y el consecuente aumento de la longevidad, dando como resultado el envejecimiento de la población.

En Latinoamérica y el Caribe la población de 60 años y más está aumentando sostenidamente, es un proceso generalizado de envejecimiento de las estructuras demográficas que lleva a un aumento, tanto en el número de personas adultas mayores, como el peso de esta población en la población total (CELADE, 2002, p.9). 
Lo anterior lleva a señalar que no solo en Latinoamérica y el Caribe sino también en Panamá la economía crece pero no mejora las múltiples desigualdades, de allí que es necesario atender a la población adulta mayor preparando sistemas de salud adecuados con condiciones que contribuyan a proteger la dignidad de esta población vulnerable, evitando que la sociedad margine a las personas que tenga características como la vejez.

Existen muchos organismos internacionales que abordan el tema de envejecimiento, a continuación se señalan algunos de ellos:

La Convención Interamericana sobre la Protección de los Derechos Humanos de las Personas Mayores define a las personas mayores como aquellas de 60 años o más, salvo que la ley interna del país determine una edad base menor o mayor, siempre que esta no sea superior a los 65 años (OEA, 2015). También la Organización Mundial de la Salud (OMS) lo define como:

Proceso fisiológico que comienza en la concepción y ocasiona cambios en las características de las especies durante todo el ciclo de la vida; esos cambios producen una limitación de la adaptabilidad del organismo en relación con el medio. Los ritmos a que estos cambios se producen en los diversos órganos de un mismo individuo o en distintos individuos no son iguales (OMS, 2016, p.15).

A medida que el nivel de ingreso de las personas mayores disminuye, las enfermedades y la muerte son las realidades que experimentan, lo que se trata es mantener personas sanas que contribuyan al bienestar de la familia y de la comunidad.

Autores plantean que el año de inicio de la vejez puede fijarse por la vía legal mediante las legislaciones de cada país, así como mediante las instituciones que se ocupan de dicha población y la que se genera en el medio académico. En Panamá, el Instituto Nacional Estadística y Censo considera a los adultos mayores a partir de los 60 años de edad, haciendo referencia a esta población con uso indistinto en los siguientes conceptos: envejecimiento, población envejecida, edad avanzada y adulto mayor (INEC,2010). Un adulto mayor es un individuo que se encuentra en una etapa de la vida, la que sigue tras la adultez y que antecede al fallecimiento de la persona, en esta etapa el cuidado no es sólo material, es multidimensional, toda política pública debe tener un impacto que busque 
atender a este grupo de personas que día a día se incrementa. Es precisamente durante esta fase que el cuerpo y las facultades cognitivas de las personas se van deteriorando y existe una mayor dependencia. El Estado en sus distintos niveles debe crear conciencia entre las instituciones involucradas con la creación de estrategias a mediano y largo plazo que atiendan a esta población.

Si se analizan las diferencias en el comportamiento demográfico entre regiones resultan significativas. Por ejemplo: En la actualidad, uno de cada 5 europeos y uno de cada 20 africanos tienen 60 y más años, (ONU, 2019). De igual manera, y como el ritmo de envejecimiento en los países en desarrollo es más rápido que en los países desarrollados, los primeros (por ejemplo, los de América Latina y el Caribe) tendrán menos tiempo para adaptarse a las consecuencias del envejecimiento de la población. Estas consecuencias serán múltiples; así, el impacto del envejecimiento de la población es cada vez más evidente en el índice de dependencia de las personas mayores (número de personas de 60 y más años en relación con las que están en edad de trabajar). Esta mayor presencia relativa de personas mayores respecto de otros grupos etarios, y las manifestaciones y consecuencias múltiples del proceso de envejecimiento, constituyen temas centrales y urgentes del desarrollo social a escala mundial. Por ejemplo, en países como: Canadá (82.2 años), Chile (80.5), Costa Rica (79.6), Estados Unidos (79.3), Cuba (79.1) y Panamá (77.8) ocupan los primeros 6 puestos (OPS, 2017).

Al envejecer aumenta el riesgo de enfermarse y en estos momentos a causa del COVID-19 el riesgo se incrementa para el adulto mayor, sobre todo los que presentan enfermedades de base, al contagiarse requerirán atenciones hospitalarias especiales, donde se sugiere tomar medidas y preservar la vida del adulto mayor.

Desde el año de 1982 que se realizó la Primera Asamblea Mundial sobre el Envejecimiento, los resultados muestran la preocupación de la comunidad internacional acerca del tema y una de las medidas era proteger a la población de mayor edad, la Asamblea General de la ONU proclamó el año 1999 Año Internacional de las Personas de Edad. El Día Internacional de las Personas de Edad se celebra el 1 de octubre de cada año y la creación de leyes a favor de esta población hace que en diversos países se celebre este 
día con diversas actividades de comunicación, salud preventiva y apoyo general. La segunda asamblea mundial celebrada en el año 2002 promueve el diseño de políticas sobre el envejecimiento y en dicha Asamblea salió una declaración política, en la cual los 160 Estados asistentes se comprometieron a adoptar medidas de incorporar eficazmente el Envejecimiento en las estrategias, políticas y acciones socioeconómicas, teniendo presente que las políticas concretas variarán en función de las condiciones de cada país.

La Tercera Conferencia Regional Intergubernamental sobre Envejecimiento en América Latina y El Caribe se realizó en el año 2012 en San José de Costa Rica, con el fin de identificar las acciones claves relacionadas con la protección social de los derechos humanos (civiles, políticos, económicos, sociales y culturales), para garantizar las condiciones de calidad de vida y dignidad de los Adultos Mayores. Todas estas asambleas y reuniones internacionales tratan de destacar que el envejecimiento es un compromiso de todos que se deben hacer cambios profundos en los sistemas de salud, en las políticas sociales que implemente cualquier Estado, de manera que los beneficios que ha generado la globalización lleguen a todas las personas. Hay un grupo de países que experimentarán incremento en sus mayores de 60 años en las próximas décadas y entre ellos se encuentra Panamá.

\section{Delimitación conceptual}

Según la CEPAL, la condición socioeconómica de los adultos mayores de la región está deteriorada por la falta de sistemas de seguridad social de amplia cobertura al momento del retiro, por la debilidad y fragilidad de los sistemas de salud para enfrentar sus necesidades y por la falta de políticas y de mecanismos de integración social e intergeneracional (CEPAL, 2000). Envejecer se ha convertido en un hecho común al alcance de un mayor número de personas, pero con ello surgen dinámicas socioeconómicas que se traducen en pérdida de autonomía, trabajo e ingresos, deterioro de la salud y exclusión en la toma de decisiones, entre otras. La vejez en el país no debe considerarse como una estadística, implica una problemática social, económica y política, toda la población tiene derechos y los adultos mayores el derecho a tener una vejez activa y ser protagonistas en la sociedad. 
La prolongación de la vida humana es un éxito de la sociedad moderna, por los avances logrados en la sociedad, pero aparecen problemáticas de salud, el incremento de las enfermedades crónicas y degenerativas, mayor dependencia y la carencia en las instituciones públicas de recursos en los servicios de salud, entre otros. Un informe del Instituto Nacional de Estadística y Censo de la Contraloría General de la República, explica que Panamá ha pasado de un crecimiento alto (una tasa de 3.17 entre 1911 y 1920) a un crecimiento más lento (una tasa anual promedio de 2.00 entre 1990 y el 2000; y de 1.84 entre 2000 y 2010) y esta situación se mantendrá durante los próximos 25 años, como consecuencia directa de la disminución de la fecundidad a nivel nacional (INEC,2010). Es necesario la identificación de propuestas para formular políticas públicas y promover el fomento de acciones de la sociedad civil en torno a los temas del envejecimiento, obligando revisar conceptos como "vulnerabilidad social" y la identificación de las personas adultas mayores como grupos vulnerables son el primer desafío.

Existen dificultades metodológicas que impiden una clara medición de los grupos vulnerables, particularmente los adultos mayores. Los estudios de Fabiana del Popolo, miden el grado de bienestar de las personas mayores, a través del Índice de Bienestar para el Adulto Mayor, (IBAM), que se apoyó en las dimensiones básicas usadas por el PNUD para construir el Índice de Desarrollo Humano (IDH) (Del Popolo, 2001). El término vulnerable como situación inestable de riesgo se refiere a las desventajas que tiene la población adulta mayor ya sea por enfermedad, falta de recursos o que están afectados en su bienestar personal o moral, es decir, en cuanto a la fragilidad humana.

\section{Envejecimiento en Panamá}

En Panamá, en las últimas cinco décadas, el grupo de 60 y más años de edad no presentaba cambios porcentuales significativos, durante los años 1960 y 1970 esta población mantuvo una proporción estable 5.6 y 5.7 por ciento, respectivamente, pero según el Informe Envejecimiento Demográfico en Panamá período 1960-2050, a partir del 2010 la proporción de este grupo de edad se incrementa alcanzando un 9.7 por ciento, superando el parámetro del 7\% establecido por Naciones Unidas, y se espera que hacia el 
2050 represente el 24 por ciento de la población total, inclusive su peso relativo será mayor que el del grupo de 0 a 14 años (CEPAL, 2014).

Según las proyecciones del Instituto Nacional de Estadística y Censo, la población de Panamá alcanzó en el año 2017, cerca de cuatro millones 100 mil habitantes, donde el $50.2 \%$ son hombres y 49.8\% mujeres. Para el año 2020 la población es de 4,278,500 habitantes manteniendo el \% de hombres y mujeres del año 2017. Además, para el año 2020 la población de adultos mayores es de 532,039 personas que corresponde al 12.1\% del total de la población, hubo un incremento del $0.70 \%$ si se compara con la población estimada para el año 2017. Ver tabla 1.

Tabla 1. Población de Panamá por sexo y de 60 años y más: año 2017 y 2020

\begin{tabular}{l|c|c|c|c|c|c}
\hline $\begin{array}{c}\text { Grupo } \\
\text { de } \\
\text { edad }\end{array}$ & \multicolumn{7}{|c}{ Número de personas } \\
\hline & \multicolumn{2}{|c|}{ Total } & \multicolumn{2}{c}{ Hombres } & \multicolumn{2}{c}{ Mujeres } \\
\hline & 2017 & 2020 & 2017 & 2020 & 2017 & 2020 \\
\hline Total & $4,098,135$ & $4,278,500$ & $2,056,085$ & $2,144,802$ & $2,042,050$ & $2,133,698$ \\
\hline $\begin{array}{l}60 \\
\text { más }\end{array}$ & 469,936 & 532,039 & 221,967 & 250,908 & 247,969 & 281,131 \\
\hline
\end{tabular}

Fuente: Elaboración a partir de INEC, estimación y proyecciones de población total, según sexo y edad al 1 de julio 2010-2020.

En el año 2017 según estimaciones la población de 60 años y más correspondió el $11.5 \%$ del total de la población en el país, teniendo un peso de $11.8 \%$ en las provincias y $6.5 \%$ en las comarcas indígenas, aquí se observa que la participación \% según territorio se ubica en las provincias de Los Santos (20.7\%), Herrera (17.2\%) y Veraguas (14.2\%). Ver tabla 2.

Tabla 2. Población de 60 años y más, en la República de Panamá, según territorio año: 2017

\begin{tabular}{l|r|r}
\hline \multirow{2}{*}{ Territorio } & Número de personas & Participación \% \\
\cline { 2 - 3 } & $\mathbf{6 0}$ y más & $\mathbf{6 0}$ y más \\
\hline \multicolumn{1}{c|}{ República de Panamá } & 469,936 & 11.5 \\
\hline Bocas del Toro & 9,319 & 5.6 \\
\hline Coclé & 32,746 & 12.5 \\
\hline Colón & 26,692 & 9.4 \\
\hline Chiriquí & 62,420 & 13.7 \\
\hline Darién & 5,570 & 10.0 \\
\hline
\end{tabular}




\begin{tabular}{|c|c|c|}
\hline Herrera & 20,391 & 17.2 \\
\hline Los Santos & 19,759 & 20.7 \\
\hline Panamá & 240,852 & 11.2 \\
\hline Veraguas & 35,038 & 14.2 \\
\hline Guna Yala & 5,136 & 11.6 \\
\hline Emberá & 955 & 7.8 \\
\hline Ngabe Buglé & 11,058 & 5.3 \\
\hline \multicolumn{3}{|c|}{ Resumen Provincias y Comarcas } \\
\hline Provincias & 452,787 & 11.8 \\
\hline Comarcas Indígenas & 17,149 & 6.5 \\
\hline
\end{tabular}

Fuente: Elaboración a partir de INEC, estimación y proyecciones de población total, según grupo de edad, al 1 de julio 2017.

En Panamá, en la población adulta mayor hay más mujeres que presentan mayor longevidad y en los hombres la tasa de mortalidad es alta, a pesar de que las mujeres realizan una doble carga de trabajo y la inequidad política y social. En las provincias de Veraguas, la comarca Emberá Wounaan y Bocas del Toro, la cifra de hombres adultos mayores es alta, este indicador muestra el número de mujeres por cada cien hombres, cuando el valor es mayor a 100 hay más mujeres que hombres y viceversa. Ver tabla 3.

Tabla 3. Índice de feminidad de la población de 60 años y más, en la República de Panamá, según territorio: año 2017

\begin{tabular}{l|r|r}
\hline \multicolumn{1}{c|}{ Territorios } & \multicolumn{1}{|c|}{ Total (\%) } & \multicolumn{1}{c}{ 60 y más (\%) } \\
\hline República de Panamá & 99.3 & 111.7 \\
\hline Bocas del Toro & 95.5 & 74.7 \\
\hline Coclé & 95.9 & 102.1 \\
\hline Colón & 97.4 & 103.1 \\
\hline Chiriquí & 98.9 & 108.1 \\
\hline Darién & 85.1 & 67.4 \\
\hline Herrera & 99.2 & 113.8 \\
\hline Los Santos & 99.0 & 112.9 \\
\hline Panamá & 101.2 & 120.6 \\
\hline Veraguas & 92.6 & 95.8 \\
\hline Guna Yala & 106.1 & 125.3 \\
\hline Emberá & 89.5 & 82.3 \\
\hline Ngabe Bugle & 103.1 & 109.4 \\
\hline Resumen Provincias y & & \\
Comarcas Indígenas & & 111.7 \\
\hline Provincias & 6.5 & 112.1 \\
\hline Comarcas Indígenas & & \\
\hline
\end{tabular}

Fuente: Elaboración a partir de INEC, estimaciones y proyecciones de la población total, según sexo y edad, al 1 de julio 2017. 
La población envejecida seguirá aumentando en las próximas décadas y ante este escenario se requiere conocer las condiciones de vida de los adultos mayores, el aumento de la población envejecida traerá consigo demandas específicas de seguridad social, económicas, de trabajo e ingresos y sobre todo los servicios de salud que en esta etapa son cruciales, se incrementan las enfermedades y los cuidados. Para las mujeres su esperanza de vida en promedio es cinco años más que los hombres, es decir 79 años frente a 74 años, (INEC, 2010).

La hormona antioxidante (estrógenos), que produce de forma natural la mujer, hace que sean menos propensas a padecer enfermedades como alzhéimer, demencia, párkinson, accidentes cerebrovasculares, infartos y fallas renales (OPS, 2017). La población adulta mayor al demandar estos servicios requerirá que el personal que atiende en estas instituciones se sensibilice de manera que todo servicio sea de calidad hacia esta población.

Si se analiza a nivel del país, las expectativas de vida varían, siendo desfavorable en las regiones comarcales. En las provincias de Los Santos, Herrera, Panamá y Chiriquí, por ejemplo, la esperanza de vida es en términos generales de 78 años, un año más que el promedio (INEC, 2010). No ocurre lo mismo, cuando se pasa a evaluar las cifras en las regiones indígenas y se compara con la media nacional, la edad promedio desciende ocho años, como es el caso de la comarca Emberá Wounaan, en donde el promedio de vida es de 69 años; en la región Ngäbe Buglé es de 71 años, y en la comarca Guna Yala, de 73 años.

A medida que aumentan las limitaciones propias del envejecimiento, se incrementa la dependencia y a medida que la tasa se incrementa, aumenta la carga que supone la parte productiva que la población debe mantener, según las estimaciones del INEC este indicador se incrementa de forma progresiva en la población de adultos mayores, que obliga a que aquellas instituciones $u$ organizaciones creadas tengan presupuestos para atender a esta población. Para el año 2017, la tasa de dependencia total a nivel nacional se ubicó en 61.9\% personas por cada 100 personas en edades económicamente activas, la vejez es de 18.6\%, la tasa de dependencia de adultos mayores más altas se concentra en Los Santos en 34.2\%, Herrera $28.1 \%$ y Veraguas $24.7 \%$. Ver tabla 4. 
Tabla 4. Tasas de dependencia y de vejez, en la República de Panamá, año 2017

\begin{tabular}{l|r|r}
\hline \multicolumn{1}{c|}{ Territorio } & \multicolumn{1}{|c}{ Total (\%) } & Población adulta mayor (\%) \\
\hline República de Panamá & 61.9 & 18.6 \\
\hline Bocas del Toro & 77.1 & 10.0 \\
\hline Coclé & 65.0 & 20.7 \\
\hline Colón & 67.5 & 15.7 \\
\hline Chiriquí & 72.2 & 23.5 \\
\hline Darién & 72.8 & 17.3 \\
\hline Herrera & 63.4 & 28.1 \\
\hline Los Santos & 65.2 & 34.2 \\
\hline Panamá & 53.1 & 17.2 \\
\hline Veraguas & 73.7 & 24.7 \\
\hline Guna Yala & 99.9 & 23.2 \\
\hline Emberá & 82.5 & 14.2 \\
\hline Ngabe Bug & 94.5 & 10.3 \\
\hline Resumen provincias y comarcas & & 18.9 \\
\hline Provincias & 60.0 & 12.6 \\
\hline Comarcas Indígenas & 94.7 & \\
\hline
\end{tabular}

Fuente: Elaboración a partir de INEC, estimaciones y proyecciones de la población total, según sexo y edad, al 1 de julio 2017.

Expertos en la salud consideran que el hecho de que la esperanza de vida haya aumentado se debe a que se tienen fármacos y equipos médicos que han ayudado a brindar mejores tratamientos a la población, ellos sugieren dirigir los servicios de salud hacia la atención primaria e impulsar estilos de vida saludables, para que las personas consuman alimentos adecuados, hagan ejercicio y acudan con regularidad a sus visitas médicas preventivas. Se promueve el Censo de Salud Preventivo, como una herramienta para conocer el estado de salud de las personas y así brindarles la atención oportuna.

El INEC, señala que la población de Panamá tendrá más de 4.8 millones de habitantes en el año 2030, es decir, un incremento del $21.6 \%$ si se compara con el año 2015. En el año 2050 la población será aproximadamente de 5.6 millones de personas, habrá un aumento de $41.5 \%$ al compararla al año 2015 y el peso de la población adulta mayor se incrementará llegando al 24\% en el año 2050 (INEC, 2010). El proceso de transición demográfica pone en evidencia dos fenómenos que se requieren analizar: el envejecimiento poblacional y el fin del bono demográfico. Panamá se encuentra en la fase de plena transición y las características son de natalidad moderada a mortalidad moderada o baja. Ver tabla 5. 
Tabla 5. Población de Panamá de 60 y más y composición etaria: años 2015 a 2050

\begin{tabular}{l|c|c|c}
\hline \multicolumn{3}{|c|}{ No de Personas } & Composición Etaria (\%) \\
\hline Año & Total & 60 y más & 60 y más \\
\hline 2015 & $3,975,404$ & 433,430 & 10.9 \\
\hline 2020 & $4,278,500$ & 532,039 & 12.4 \\
\hline 2025 & $4,565,559$ & 651,972 & 14.3 \\
\hline 2030 & $4,834,846$ & 787,845 & 16.3 \\
\hline 2040 & $5,306,666$ & $1,079,795$ & 20.3 \\
\hline 2050 & $5,625,442$ & $1,351,367$ & 24.0 \\
\hline
\end{tabular}

Fuente: Elaboración a partir de INEC, estimaciones y proyecciones de la población total, según sexo y edad, al 1 de julio 2015-2030.

La vida adulta mayor tiene fuertes repercusiones en las condiciones de salud mental, que se pueden agravar por la pobreza, la indiferencia de la familia, el maltrato físico y psicológico, al igual que la soledad. En la tabla 6 se observa el indicador tasa de dependencia, situación ya señalada donde los adultos mayores aumentan en forma progresiva. El cuidado de las personas mayores es una responsabilidad, urge que las instituciones vinculadas elaboren planes coherentes e integrados con un renglón de seguimiento que atiendan esta problemática.

Tabla 6 Tasa de dependencia total y adulta mayor: año 2015-2050

\begin{tabular}{|c|c|c|}
\hline Año & Total & 60 y más años de edad \\
\hline 2015 & 62.2 & 17.7 \\
\hline 2020 & 61.8 & 20.1 \\
\hline 2025 & 62.5 & 23.2 \\
\hline 2030 & 64.0 & 26.7 \\
\hline 2040 & 68.7 & 34.3 \\
\hline 2050 & 74.2 & 41.9 \\
\hline
\end{tabular}

Fuente: Elaboración a partir de INEC, estimaciones y proyecciones de la población total, según sexo y edad, al 1 de julio 2015-2030.

Al analizar la salud social de la población adulta mayor, se evidencia que los pobres e indígenas son los más vulnerables, tienen menor acceso a la atención de salud y la ubicación de la población a nivel nacional también incide en los servicios básicos. Estudios demuestran que los adultos(as) mayores más vulnerables son los de 75 y más años, ya que son quienes más riesgos de enfermedad presentan, en Panamá el mayor porcentaje de adultos(as) mayores de esta edad pertenece a las mujeres, las cuales a su vez forman el mayor grupo 
de viudas A medida que el individuo envejece tiene mayor riesgo de padecer una enfermedad, la cual tiende a ser crónica e incapacitante. Según el último censo, el 25.1\% de los hogares panameños tienen como jefe a una persona de 60 y más años, que viven especialmente en los hogares nucleares biparentales con o sin hijos (INEC, 2010).

Al revisar se encuentran en el país 35 instituciones de Protección Familiar y de Atención a la población adulta mayor, de las cuales, 12 son subsidiadas por el Estado a través del Ministerio de Desarrollo Social (MIDES), y 23 son de carácter privado. Para el año 2020, en las instituciones subsidiadas por el Estado hay 843 adultos(as) mayores: 491 hombres y 352 mujeres (MIDES, 2019). En cuanto a la población atendida en las instituciones privadas hay un total de 451 personas: 235 del sexo masculino y 216 del sexo femenino. EI MIDES lleva a cabo el programa 120 a los 65 que beneficia a 125 mil personas, por un monto de 45.2 millones de dólares (MIDES, 2020).

La vida y la salud constituyen Derechos Humanos esenciales para que las personas alcancen su máximo desarrollo individual y un requisito para la construcción de sociedades justas y democráticas, no se puede hablar de crecimiento económico cuando la Comisión Económica para América Latina y el Caribe (CEPAL) estimó que la pobreza en Panamá aumentará un 2,9\%, como resultado de la crisis generada por la pandemia Covid-19, pasando de $14,6 \%$ a $17.5 \%$ (CEPAL, 2020). Todos los derechos humanos son universales, indivisibles e interdependientes, pero la fuente de todos estos derechos es la dignidad de la persona humana y su base está en satisfacer integral y armónica sus necesidades y desde esta perspectiva se debe procurar atender a los adultos mayores que no son una carga, son personas con dignidad y con derecho a un estándar de vida adecuado.

La mayor participación de las personas mayores se concentra en el sector primario de la economía (46.3\%). La participación de los hombres se ubica fundamentalmente en actividades silvoagropecuarias, con marcado dinamismo en el área rural. En las mujeres (68\%) su mayor participación está en los servicios domésticos, comunitarios, sociales y personales (PNUD, 2020). 


\section{Políticas públicas}

La Constitución Nacional en su artículo 59 dispone la protección y atención que el Estado debe brindar a la familia en general y destaca un apoyo especial a la población adulta mayor, principalmente en aquella que se encuentra en estado de vulnerabilidad. El artículo 109 de la Carta Magna hace referencia al papel esencial del Estado que entre otros aspectos debe velar por la salud y el bienestar de toda la población de la República.

La ley 36 del 2 de agosto de 2016, está dirigida a proteger los derechos de las personas mayores de 60 años, sean nacionales o extranjeros residentes, esta norma promueve la promoción de planes y programas educativos y de capacitación para la población adulta mayor. Esta Ley establece el marco normativo de manera que se garantice los derechos que aparecen en la Constitución Política de la Republica a las personas adultas mayores. En su artículo 2 tiene entre otros objetivos:

a) Garantizar la defensa de los derechos de las personas adultas mayores para la satisfacción de sus necesidades de salud, educación, alimentación, vivienda, vestuario, seguridad, esparcimiento, trabajo y atención social.

b) Desarrollar procesos de consulta para las personas adultas mayores en la formación de políticas públicas que las afecten.

Al revisar el Código de la Familia el artículo 569, destaca la responsabilidad del Estado en desarrollar políticas sociales de prevención, protección y promoción del bienestar general de la familia en su conjunto y de los grupos vulnerables en particular, a pesar de contar con estas normas y leyes establecidas desde muchos años, el país mantiene una deuda con esta población.

La transición demográfica es un factor clave para identificar la necesidad de establecer políticas públicas para las personas de 60 y más años y se observa políticas débiles o nula respecto de normativas legales en favor de la población que envejece. Todo desafío para mejorar la calidad de vida de los adultos mayores reclama una política estatal y la acción de toda la sociedad.

Si bien cada realidad nacional permite definir los marcos contextuales de las políticas nacionales, vale la pena destacar los compromisos adoptados en foros internacionales y 
rescatar los criterios políticos fundamentales. A continuación, se mencionan varios criterios que pueden ayudar al diseño de principios y objetivos desde la perspectiva de la pobreza y la vulnerabilidad de los adultos mayores:

a) La política dirigida a las personas adultas mayores debe formar parte integrante de un desarrollo humano sostenible.

b) Toda política que busque enfrentar los problemas del envejecimiento debe considerar enfoques multidisciplinarios.

c) La política debe promover la solidaridad entre generaciones con el objetivo de construir y desarrollar estereotipos culturales que valoren el aporte de las personas mayores y la transmisión de sus habilidades y experiencias a las nuevas generaciones.

d) Las políticas sobre envejecimiento deben tener una capacidad convocatoria a la participación, con el objetivo de incorporar a los adultos mayores, grupos sociales y otros agentes de desarrollo que trabajan en el tema del envejecimiento (ONG, iglesias, universidades) en el diseño y ejecución de programas.

e) Las políticas públicas deben ser diseñadas propiciando la vigencia plena de los derechos fundamentales de las personas mayores y poner acento en el rescate de los derechos de grupos de adultos mayores muy vulnerables (étnicos, discapacitados, maltratados, etc.).

Una revisión general señala que aún existen muchos países donde no hay un marco legal específico para las personas mayores y en otros casos existen leyes que no funcionan y los esfuerzos están disgregados en instituciones públicas que con poco o muchos recursos no dan respuestas a esta población, de allí que es un desafío construir alianzas efectivas con entidades públicas y con la capacidad de movilizar recursos desde las realidades nacionales.

Desde una perspectiva general, las políticas públicas deben construirse con responsabilidad identificando las acciones a los problemas críticos y en este caso de las personas adultas mayores, que estas acciones busquen incentivar positivamente su participación en la vida política, económica, social y cultural, de manera que hagan efectiva su participación en la sociedad y enfrenten sus propios desafíos mediante sus propias organizaciones. 
El aumento de la esperanza de vida debe ser motivo de preocupación para los responsables de formular políticas, urge crear instituciones económicas y sociales que brinden seguridad en cuanto a los ingresos, presten una adecuada atención de salud y satisfagan otras necesidades de la población que está envejeciendo. La seguridad económica de las personas mayores en Panamá es heterogénea y marcada por el género, la zona de residencia y el origen étnico, donde un quinto de la población adulta mayor carece de ingresos y se tiene una informalidad por encima del $50 \%$.

\section{Conclusiones}

- Distintos documentos plantean que la proporción de personas en edad avanzada de 60 años en Panamá comenzará a crecer a partir de 2020 y se espera que continúe su ascenso hasta alcanzar, aproximadamente, el 24\% de la población total en 2050. Esta situación aumentará en términos absolutos y relativos tanto en hombres como mujeres y la estimación que hace el INEC indica que al año 2040 sobrepasará el millón de personas. Otro aspecto es el índice de feminidad que señala que habrá más mujeres que hombres.

- Las políticas públicas aplicadas a la fecha son insuficientes en cuanto a la promoción de la salud y la mejora a infraestructuras hospitalarias. El envejecimiento de la población trae consigo cambios que impactarán la seguridad social, la economía y el sistema de salud. Además, hay pocas instituciones creadas para el cuidado de esta población.

- El presupuesto que se destina a través de las instituciones de salud no es suficiente para atender enfermedades que son la causa de muerte entre los adultos mayores como, por ejemplo: tumores malignos, enfermedades isquémicas del corazón, las cerebrovasculares y la diabetes.

- Panamá cuenta con la Ley 36 del 2 de agosto de 2016, una legislación dirigida a proteger los derechos de las personas mayores de 60 años, sean nacionales o extranjeros residentes. Pero existen muchos aspectos no desarrollados en esta Ley, por ejemplo: El Instituto para el Adulto Mayor no se ha reglamentado. 


\section{Referencias bibliográficas}

Asamblea Legislativa de la República de Panamá. (1994). Ley N³ del 17 de mayo de 1994. "Código de la familia", que comprende un conjunto de normas fundamentales concernientes a los deberes y derechos de la familia y sus diferentes componentes.

Asamblea Nacional de la República de Panamá. (2016). Ley 36 del 2 de agosto de 2016. Que establece la normativa para la protección integral de los derechos de las personas adultas mayores.

Asamblea Nacional de Legisladores de la República de Panamá. (1972). Constitución política de la República de Panamá.

CELADE. (2002). Envejecimiento y vejez en América Latina y el Caribe: Políticas públicas y las acciones de la sociedad CELADE-FNUAP (Fondo de Población de las Naciones Unidas).

CEPAL. (2000). Panorama social de América Latina, Serie LC/G. 2068-P. Vulnerabilidad demográfica en Bolivia, Ecuador, Nicaragua y Uruguay, serie LC/R. 1989. Santiago de Chile.

CEPAL. (2014). Los efectos y desafíos de la transformación demográfica en América Latina y el Caribe. Santiago de Chile

CEPAL. (2019). Primer informe regional sobre la implementación del consenso de Montevideo sobre población y desarrollo. Santiago de Chile.

CEPAL. (2020). América Latina y el Caribe ante la pandemia del COVID-19: efectos económicos y sociales, Informe Especial COVID-19, № 1, 3 de abril. Recuperado de https://repositorio.cepal.org/bitstream/handle/11362/45337/4/S2000264 es.pdf.

Del Popolo, F. (2001). Características sociodemográficas y socioeconómicas de las personas de edad en América Latina. CEPAL - CELADE. Serie 19.

INEC. (2010). Estimaciones y proyecciones de la población de la República, por provincia y comarca indígena, según sexo y edad: años 2000-30. Panamá boletín 14. Panamá.

Ministerio de Desarrollo Social. (s.f.). Memoria 2019. Recuperado de https://www.mides.gob.pa/wp-content/uploads/2020/01/Memoria2019.pdf 
OEA. (2015). Envejecimiento y derechos humanos. La convención interamericana sobre la protección de los derechos humanos de las personas mayores.

OMS. (2016). Estrategia y plan de acción mundiales sobre el envejecimiento y la salud 20162020. Hacia un mundo en el que todas las personas puedan vivir una vida prolongada y sana. (2016). Recuperado de http://apps.who.int/gb/ebwha/pdf files/WHA69/A69 R3-sp.pdf

ONU. (2014). Los derechos de las personas mayores: materiales de estudio y divulgación. Hacia un cambio de paradigma sobre envejecimiento y vejez. CEPAL 21 de octubre de 2014

ONU. (2019). Naciones Unidas. Memoria. Recuperado de https://www.mides.gob.pa/wpcontent/uploads/2020/01/Memoria2019.pdf

Organización Panamericana de la Salud / Organización Mundial de la Salud. (s.f.). Salud en las Américas. Recuperado de https://www.paho.org/salud-en-las-americas2017/?post type=post $t$ es\&p=320\&lang=es

PNUD. (2020). Impacto del covid 19 en Panamá. Análisis socioeconómico. Recuperado de https://panama.un.org/es/48599-analisis-socioeconomico-del-impacto-del-covid19-en-panama 